\title{
Optical coherence tomography of traumatic aneurysms of the internal carotid artery: report of 2 cases
}

\author{
Christoph J. Griessenauer, MD, Paul M. Foreman, MD, John P. Deveikis, MD, and \\ Mark R. Harrigan, MD
}

Department of Neurosurgery, University of Alabama at Birmingham, Alabama

\begin{abstract}
The pathophysiology of extracranial traumatic aneurysm formation has not been fully elucidated. Intraarterial optical coherence tomography (OCT), an imaging modality capable of micrometer cross-sectional resolution, was used to evaluate patients presenting with saccular traumatic aneurysms of the internal carotid artery (ICA). Two consecutive trauma patients diagnosed with saccular traumatic aneurysms of the cervical ICA, per the institutional screening protocol for traumatic cerebrovascular injury, underwent digital subtraction angiography (DSA) with OCT. Optical coherence tomography demonstrated disruption of the intima with preservation and stretching of the more peripheral layers. In 1 patient the traumatic aneurysm was associated with thrombus formation and a separate, more proximal dissection not visible on CT angiography (CTA) or DSA. Imaging with OCT indicates that saccular traumatic aneurysms may develop from disruption of the intima with at least partial preservation of the media and adventitia. This provides in vivo evidence that saccular traumatic aneurysms result from a partial arterial wall tear rather than complete disruption. Interestingly, OCT was also able to detect arterial injury and thrombi not visible on CTA or DSA.
\end{abstract}

http://thejns.org/doi/abs/10.3171/2015.1.JNS142840

KEY WORDS internal carotid artery; blunt trauma; dissecting aneurysm; traumatic aneurysm; pseudoaneurysm; traumatic cerebrovascular injury; optical coherence tomography; vascular disorders

$\mathrm{E}$ XTRACRANIAL traumatic aneurysms are found in approximately $10 \%$ of blunt traumatic cerebrovascular injuries (TCVIs). ${ }^{4}$ While these lesions are uncommon, they have been associated with a risk of thromboembolic ischemic stroke. The term "pseudoaneurysm" has been used to describe these lesions, suggesting that there is a complete disruption of the arterial wall. ${ }^{3,14,15}$ However, a postmortem histopathological study of traumatic carotid artery aneurysms found only partial disruption of the arterial wall. ${ }^{12}$ In vivo data on the pathological anatomy of traumatic aneurysms has not been reported. Intraarterial optical coherence tomography (OCT) is approved in the US for imaging of coronary arteries. Optical coherence tomography is a catheter-based optical imaging technique that provides high-resolution cross-sectional images from tissue based on tissue reflectance of near-infrared or infrared light. Image resolution with OCT reaches $8 \mu \mathrm{m}$, which is at least 10 times greater than that of any clinically available diagnostic imaging modality. ${ }^{11}$ OCT has been used to image the layers of the internal carotid artery (ICA) in human subjects. In the present report, OCT was used to assess the pathological changes in the arterial wall of saccular traumatic aneurysms.

\section{Case Reports}

Two consecutive trauma patients diagnosed with saccular traumatic aneurysms of the cervical ICA per the institutional screening protocol for TCVI were studied at a Level I trauma center.

\section{History and Examination}

In Case 1, a 21-year-old woman presented after a motor vehicle collision. Neurological examination indicated that she had a Glasgow Coma Scale (GCS) score of 6T with intact cranial nerve reflexes and symmetric withdrawal from noxious stimuli in all 4 extremities. Computed tomography of the head demonstrated skull and skull base

ABBREVIATIONS CTA = CT angiography; DSA = digital subtraction angiography; GCS = Glasgow Coma Scale; ICA = internal carotid artery; OCT = optical coherence tomography; TCVI = traumatic cerebrovascular injury.

SUBMITTED December 15, 2014. ACCEPTED January 28, 2015.

INCLUDE WHEN CITING Published online August 7, 2015; DOI: 10.3171/2015.1.JNS142840.

DISCLOSURE LightLab Imaging, Inc. (St. Jude Medical), lent the catheters and analysis system for use in the study. 
fractures, pneumocephalus, and small areas of extraaxial hemorrhage. Other injuries included extremity fractures, facial lacerations and fractures, and periorbital hematomas. Screening CT angiography (CTA) of the neck demonstrated a saccular traumatic aneurysm of the right cervical ICA. The aneurysm measured $5 \mathrm{~mm}$ in maximum depth and arose from the lateral wall of the artery. Treatment with aspirin (325 mg daily) was initiated. On hospital Day 5 she was taken to the endovascular suite for digital subtraction angiography (DSA) with OCT.

In Case 2, a 21-year-old man presented after a motor vehicle collision. On neurological examination he had a GCS score of 10T with intact cranial nerve reflexes and movements in all 4 extremities. Computed tomography of the head demonstrated skull and skull base fractures, facial fractures, and traumatic subarachnoid hemorrhage. Other injuries included pelvic fractures and multiple extremity fractures. Screening CTA of the neck demonstrated a medially projecting saccular traumatic aneurysm of the left cervical ICA. The aneurysm measured $13 \mathrm{~mm}$ in maximum depth. Treatment with aspirin (325 mg daily) was initiated. On hospital Day 9 he was taken for DSA with OCT.

\section{Optical Coherence Tomography Procedure}

During DSA, a 6-Fr guide catheter was placed in the common carotid artery. Baseline intracranial angiograms were obtained and 5000 units of intravenous heparin were administered. A 0.014-inch Synchro Standard microwire (Stryker Neurovascular) was advanced through the guide catheter, past the traumatic aneurysm, and positioned in the petrous segment of the ICA. Next, a 2.7-Fr Dragonfly OCT imaging catheter (LightLab Imaging Inc., St. Jude Medical) was advanced over the microwire and past the aneurysm. The guide catheter was then connected to a power injector. An OCT acquisition over 2.7 seconds was conducted during power-injection of contrast material to trigger the internalized automated "pull-back" method that renders catheter manipulation unnecessary during image acquisition, and to transiently clear red blood cells from the imaging area (Figs. 1 and 2). ${ }^{1}$ Image processing and data analysis were performed using a commercially available OCT system (Ilumien System, LightLab Imaging Inc., St. Jude Medical). The use of OCT as an adjunct to DSA was performed with Institutional Review Board approval.

\section{Optical Coherence Tomography Findings}

Excellent visualization of the normal arterial wall proximal and distal to the traumatic aneurysm was acquired in both cases. In Case 1, distal to the traumatic aneurysm, at the junction between the cervical and petrous ICA, there was an intramural hematoma layered between the preserved intima and media (Fig. 1A). At the level of the traumatic aneurysm there was complete disruption of the intima covered with a thrombus and stretching of media and adventitia without disruption (Fig. 1B). Proximal to the traumatic aneurysm there was evidence of injury of the intima covered with a thrombus (Fig. 1C) and a separate dissection opposite and more proximal to the traumatic aneurysm not visible on CTA or DSA (Fig. 1D).
Video 1 demonstrates the interventional procedure with the OCT sequence.

VIDEO 1. Clip showing the interventional procedure with OCT sequence in Case 1. Copyright Christoph J. Griessenauer.

Published with permission. Click here to view with Media Player. Click here to view with Quicktime.

In Case 2, at the level of the traumatic aneurysm, the intima appeared disrupted while media and adventitia were stretched (Fig. 2A and B). Neither additional injury nor thrombus formation was visible. Visual representation over the entire length of the scanned arterial segment (Fig. 2C) showed preserved architecture of the arterial wall proximal and distal to the aneurysm.

\section{Postprocedural Course}

Both patients tolerated the procedure well and were discharged to a rehabilitation facility neurologically intact.

\section{Discussion}

Intraarterial OCT is a light-based imaging modality routinely used in the coronary vasculature to provide detailed visualization of the layers of the coronary artery wall, delineate pathophysiological processes such as degree of stenosis or the presence and composition of an atheroma or thrombus, and assess stent opposition and vascular healing. ${ }^{2,8}$ OCT technology relies on near-infrared light tissue reflectance and delivers the highest resolution among currently available vascular imaging techniques.

Despite the popularity of OCT for evaluation of the coronary vasculature, there have been relatively few published applications of OCT in the cerebrovasculature. ${ }^{11,16,17} \mathrm{~A}$ recent case report described the use of OCT for evaluation of flow-limiting stenosis in a patient who developed symptoms of posterior fossa ischemia following vertebrobasilar angioplasty and stent placement. ${ }^{6}$ Others have applied the technology for in vivo assessment of arteriosclerosis and stent-artery interactions in the carotid artery. ${ }^{1,5,9,17}$ As compared with intravascular ultrasonography, OCT appears to be superior in the detection of intraluminal thrombi and neovascularization in carotid artery plaques,$^{17}$ and has proven useful in the evaluation of an intraluminal thrombus in a patient with symptomatic ICA stenosis who underwent carotid endarterectomy instead of carotid stenting in light of this finding. ${ }^{16}$

This report details the use of OCT in 2 patients with saccular traumatic aneurysms of the ICA in an attempt to further elucidate the pathophysiology of these lesions. Traumatic aneurysms are widely referred to as pseudoaneurysms, which implies a complete disruption of the arterial wall with an aneurysm dome consisting primarily of fibrin. However, a postmortem histopathological study of traumatic injury to the carotid artery indicates that most aneurysms associated with blunt trauma result from only partial disruption of the arterial wall. ${ }^{12}$

In the present report, OCT was safely performed in awake patients with saccular traumatic aneurysms of the ICA. In both cases the traumatic aneurysm was clearly visualized. While complete disruption of the intima at the proximal level of the traumatic aneurysm was observed, there appeared to be only stretching of the media and ad- 


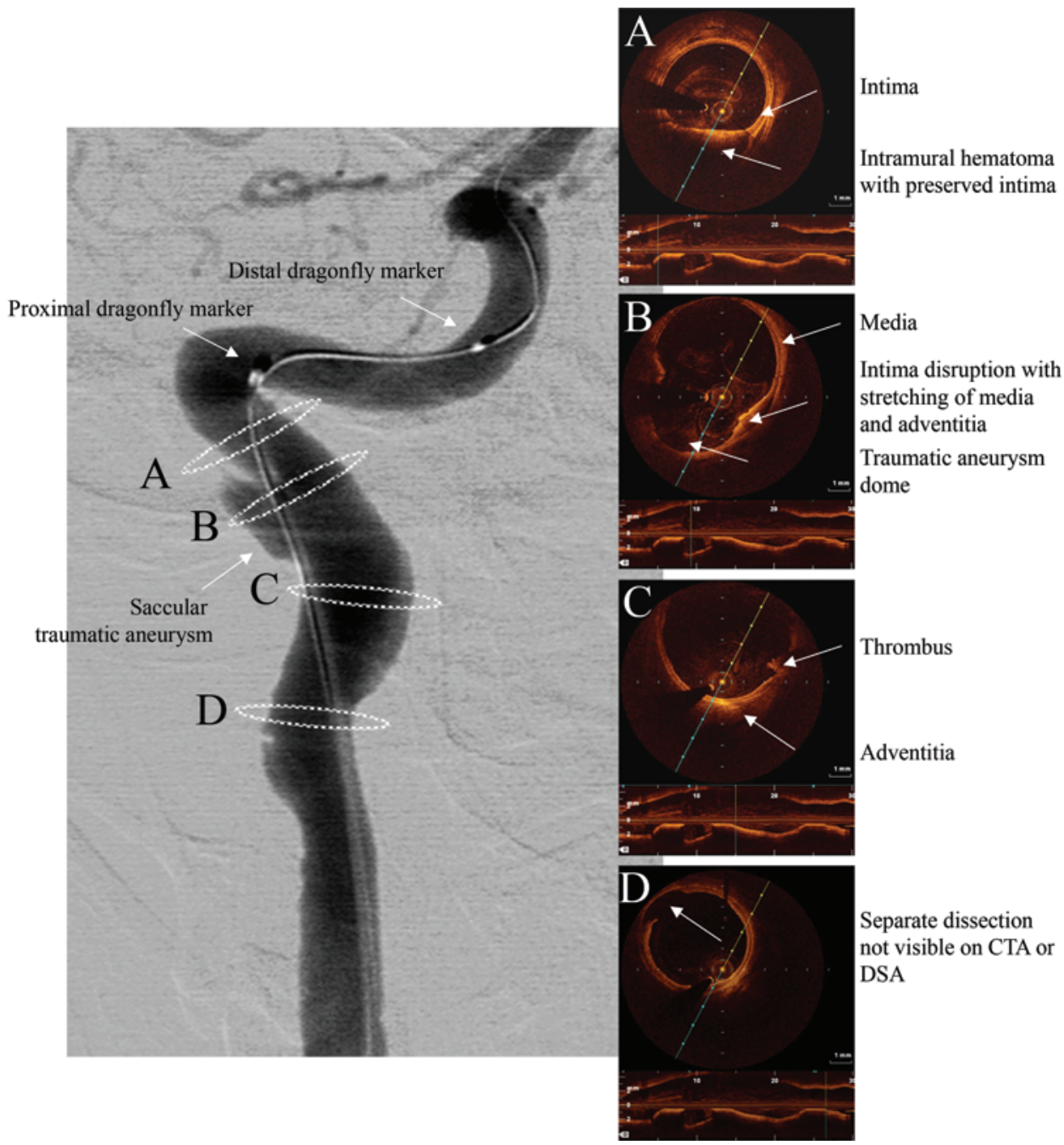

FIG. 1. Case 1. Anteroposterior projection from a digital subtraction angiogram of the right ICA shows dissection and a saccular traumatic aneurysm of the lateral wall, and the position of the Dragonfly OCT imaging catheter (left). White dashed circles on the left panel correspond to cross-sections of the OCT acquisition. Distal to the traumatic aneurysm at the junction between the cervical and petrous carotid artery there was an intramural hematoma layered between the preserved intima and media (A). At the level of the traumatic aneurysm there was complete disruption of the intima covered with a thrombus and stretching of media and adventitia without disruption (B). Proximal to the traumatic aneurysm there was evidence of injury of the intima covered with a thrombus (C) and a separate dissection not visible on CTA or DSA (D). Figure is available in color online only.

ventitia. The internal elastic lamina was not clearly visualized but presumed to be disrupted. Complete arterial wall disruption, however, was not visualized at any point along the injured vessel.

In Case 1 there was an intramural hematoma identified as layered between the intima and media near the distal end of the aneurysm. The exposed layer was covered with a thrombus and possessed imaging characteristics consistent with a platelet-rich "white" thrombus. OCT has demonstrated the ability to distinguish between a plateletrich "white" thrombus and a red blood cell and fibrin-rich "red" thrombus. "White" thrombi have been identified as low-backscattering protrusions inside the lumen while "red" thrombi exhibit high backscattering. ${ }^{10}$ There was also a disruption of the intima that was visualized on the opposite side of the artery and more proximal to the traumatic aneurysm and was not identified with DSA.
OCT in Case 2 was unremarkable aside from the traumatic aneurysm. In this patient OCT was not performed until hospital Day 9 and subtle dissections may have healed or thrombi dissolved by that time. A limitation of OCT for the evaluation of aneurysms, as applied here, is that circumferential tissue visualization is limited to only $5 \mathrm{~mm}$ from the catheter. In Case 2, the maximal extent of the aneurysm dome exceeded the 5-mm threshold, thus limiting the ability to assess the full extent of the aneurysm.

Traumatic aneurysms of the extracranial carotid and vertebral arteries occur in approximately $10 \%$ of cases of TCVI and have been associated with embolic ischemic stroke in $15 \%$ of patients. ${ }^{4}$ Two distinct traumatic aneurysm morphologies have been described (saccular and fusiform), and each type has unique clinical and imaging characterstics. ${ }^{4,7}$ Management options range from expect- 


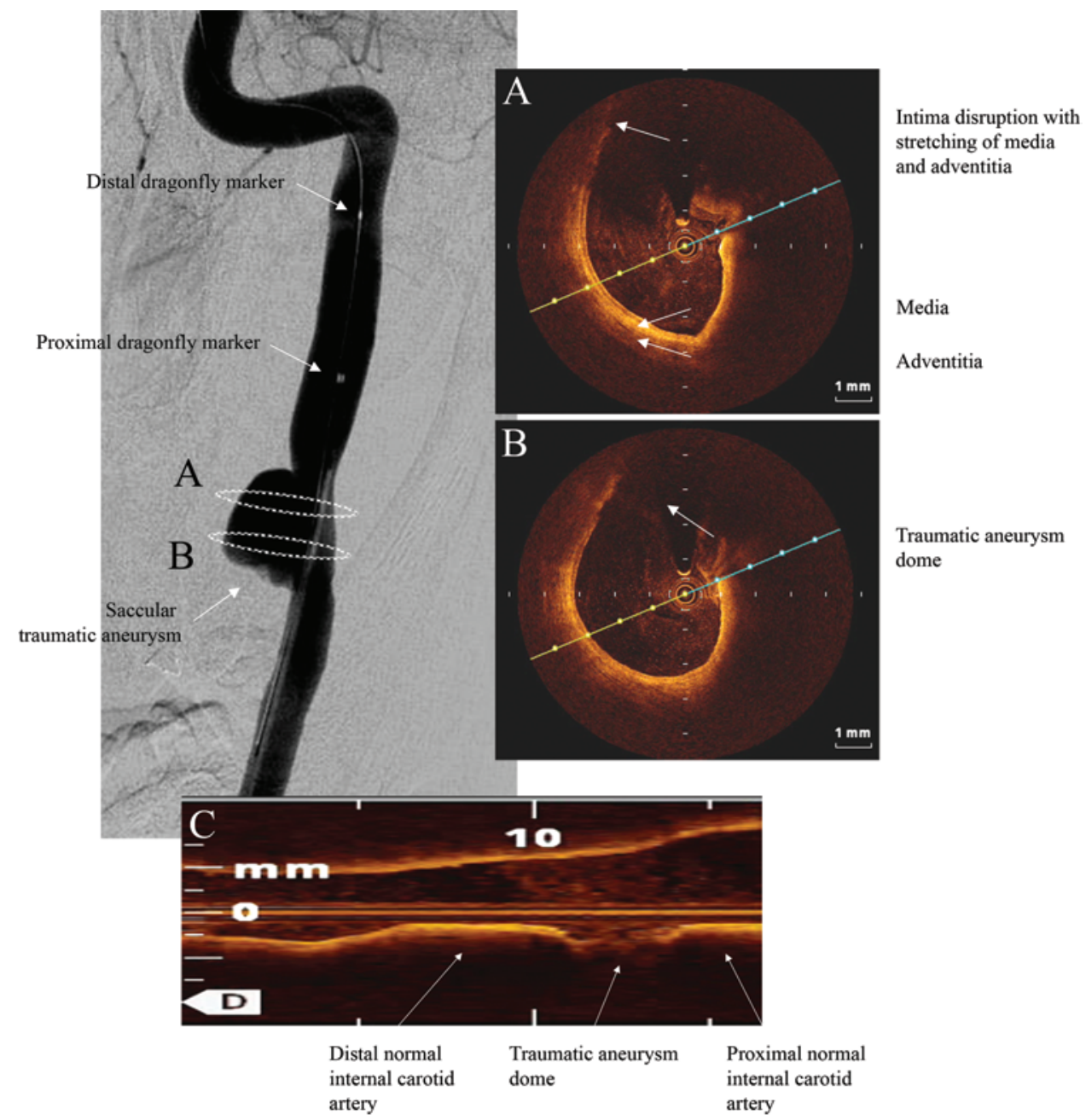

FIG. 2. Case 2. Anteroposterior projection from a digital subtraction angiogram of the left ICA shows a saccular traumatic aneurysm of the medial wall and the position of the Dragonfly OCT imaging catheter (left). White dashed circles on the left panel correspond to cross-sections of the OCT acquisition (A and B). At the level of the traumatic aneurysm the intima appeared disrupted while media and adventitia were stretched ( $A$ and $B$ ). No additional injury or thrombus formation was visible. Visual representation over the entire length of the scanned arterial segment $(\mathbf{C})$ showed preserved architecture of the arterial wall proximal and distal to the aneurysm. Figure is available in color online only.

ant management with antithrombotic therapy to endovascular repair.,13 Saccular traumatic aneurysms are more likely to enlarge over time and portend a greater risk of thromboembolic stroke as compared with fusiform traumatic aneurysms. ${ }^{4}$ Saccular traumatic aneurysms are believed to result from a tear in both the intima and the elastic laminae exposing subendothelial elements to circulating platelets and clotting factors, leading to dilation of the weakened arterial wall with an increased risk of blood stasis and thromboembolic phenomena. ${ }^{4}$ Fusiform traumatic aneurysms, in contrast, may result from stretching of the intima rather than tearing, and carry a lower risk of thromboembolism. ${ }^{4}$ The traumatic aneurysms described here were saccular and demonstrated complete disruption of the intima with preservation, but stretching, of media and adventitia, corroborating the aforementioned hypothesis. There were some characteristics of a "white" thrombus supporting an approach with antiplatelet agents as first-line treatment in Case 1. While a small subset of large saccular traumatic aneurysms will demonstrate con- tinued growth necessitating endovascular treatment, the majority of traumatic aneurysms appear to be effectively managed with antiplatelet agents and serial imaging. ${ }^{4}$

\section{Acknowledgment}

We thank LightLab Imaging Inc., St. Jude Medical for lending the catheters and analysis system for use in the study.

\section{References}

1. Attizzani GF, Jones MR, Given CA II, Brooks WH, Bezerra HG, Costa MA: Frequency-domain optical coherence tomography assessment of very late vascular response after carotid stent implantation. J Vasc Surg 58:201-204, 2013

2. Bouma BE, Tearney GJ, Yabushita H, Shishkov M, Kauffman CR, DeJoseph Gauthier D, et al: Evaluation of intracoronary stenting by intravascular optical coherence tomography. Heart 89:317-320, 2003

3. Choudhri O, Heit J, Do HM: Endovascular reconstruction of enlarging traumatic internal carotid artery pseudoaneurysm. Neurosurg Focus 37 (1 Suppl):1, 2014 
4. Foreman PM, Griessenauer CJ, Falola M, Harrigan MR: Extracranial traumatic aneurysms due to blunt cerebrovascular injury. J Neurosurg 120:1437-1445, 2014

5. Given CA II, Attizzani GF, Jones MR, Ramsey CN III, Brooks WH, Costa MA, et al: Frequency-domain optical coherence tomography assessment of human carotid atherosclerosis using saline flush for blood clearance without balloon occlusion. AJNR Am J Neuroradiol 34:1414-1418, 2013

6. Given CA, Ramsey CN, Attizzani GF, Jones MR, Brooks WH, Bezerra HG, et al: Optical coherence tomography of the intracranial vasculature and Wingspan stent in a patient. J Neurointerv Surg 7:e22, 2015

7. Griessenauer CJ, Foreman PM, Shoja MM, Kicielinski KP, Deveikis JP, Walters BC, et al: Carotid And Vertebral Injury Study (CAVIS) technique for characterization of blunt traumatic aneurysms with reliability assessment. Interv Neuroradiol 21:255-262, 2015

8. Guagliumi G, Sirbu V: Optical coherence tomography: high resolution intravascular imaging to evaluate vascular healing after coronary stenting. Catheter Cardiovasc Interv 72:237-247, 2008

9. Jones MR, Attizzani GF, Given CA II, Brooks WH, Costa MA, Bezerra HG: Intravascular frequency-domain optical coherence tomography assessment of atherosclerosis and stent-vessel interactions in human carotid arteries. AJNR Am J Neuroradiol 33:1494-1501, 2012

10. Kume T, Akasaka T, Kawamoto T, Ogasawara Y, Watanabe N, Toyota E, et al: Assessment of coronary arterial thrombus by optical coherence tomography. Am J Cardiol 97:17131717, 2006

11. Mathews MS, Linskey ME: Neuroendovascular optical coherence tomography imaging for cerebrovascular disease: current concepts and clinical potential. Curr Cardiol Rep 14:647-650, 2012

12. Moar JJ: Traumatic rupture of the cervical carotid arteries: an autopsy and histopathological study of 200 cases. Forensic Sci Int 34:227-244, 1987

13. Pham MH, Rahme RJ, Arnaout O, Hurley MC, Bernstein RA, Batjer HH, et al: Endovascular stenting of extracranial carotid and vertebral artery dissections: a systematic review of the literature. Neurosurgery 68:856-866, 2011

14. Wang W, Li MH, Li YD, Gu BX, Wang J, Zhang PL, et al: Treatment of traumatic internal carotid artery pseudoaneurysms with the Willis covered stent: a prospective study. J Trauma 70:816-822, 2011
15. Yeh C, Lin MSF, Chiu M, Chen CH, Pai Y: Endovascular treatment of a huge cervical carotid artery pseudoaneurysm with Wallgraft prosthesis. Ann Vasc Surg 25:265.e1-265.e3, 2011

16. Yoshimura S, Kawasaki M, Hattori A, Nishigaki K, Minatoguchi S, Iwama T: Demonstration of intraluminal thrombus in the carotid artery by optical coherence tomography: technical case report. Neurosurgery 67:onsE305, 2010

17. Yoshimura S, Kawasaki M, Yamada K, Enomoto Y, Egashira Y, Hattori A, et al: Visualization of internal carotid artery atherosclerotic plaques in symptomatic and asymptomatic patients: a comparison of optical coherence tomography and intravascular ultrasound. AJNR Am J Neuroradiol 33:308313,2012

\section{Author Contributions}

Conception and design: Griessenauer, Harrigan. Acquisition of data: Griessenauer, Harrigan. Analysis and interpretation of data: all authors. Drafting the article: Griessenauer. Critically revising the article: all authors. Reviewed submitted version of manuscript: Griessenauer, Harrigan. Approved the final version of the manuscript on behalf of all authors: Griessenauer. Statistical analysis: Administrative/technical/material support: Griessenauer, Harrigan. Study supervision: Griessenauer, Harrigan.

\section{Supplemental Information Video}

Video 1, Media Player. http://mfile.akamai.com/21490/wmv/ digitalwbc.download.akamai.com/21492/wm.digitalsource-naregional/jns14-2840_video_1.asx.

Video 1, Quicktime. http://mfile.akamai.com/21488/mov/ digitalwbc.download.akamai.com/21492/qt.digitalsource-global/ jns14-2840_video_1.mov.

\section{Correspondence}

Christoph J. Griessenauer, Beth Israel Deaconess Medical Center, Neurosurgery, Department of Surgery, Lowry Medical Office Building, Ste. 3B, 110 Francis St., Boston, MA 02215. email: christoph.griessenauer@gmail.com. 